\title{
IMPORTANCE OF NATURAL COLORED FOODS IN OUR DAILY DIET
}

\author{
Dr Jyoti Jagwani
}

Associate Professor (Home Sc Dept.)

Maharani Laxmi Bai Govt Girls College, Kila Maidan, Indore (M P)

Colors are not only beautiful to look at. They also affect our well being. By applying the correct color to a specific problem area within our bodies, we can influence our well being. This can also be achieved via the color of our food. The basic rule is: a little bit of everything. If we eat a colorful variety, we will be taking in all the most important nutrients necessary for a smooth functioning of all body functions. This makes one healthy and fit.

Our bodies are marvelous. They are designed in a way that they take up the color we are deficient in and excrete those not required. Fruits and vegetables contain hundreds of colorful phytochemicals that act as antioxidant, which help to mop up potentially harmful molecules called free radicals before they get a chance to damage cells.

Research shows that phytochemicals in fruits and

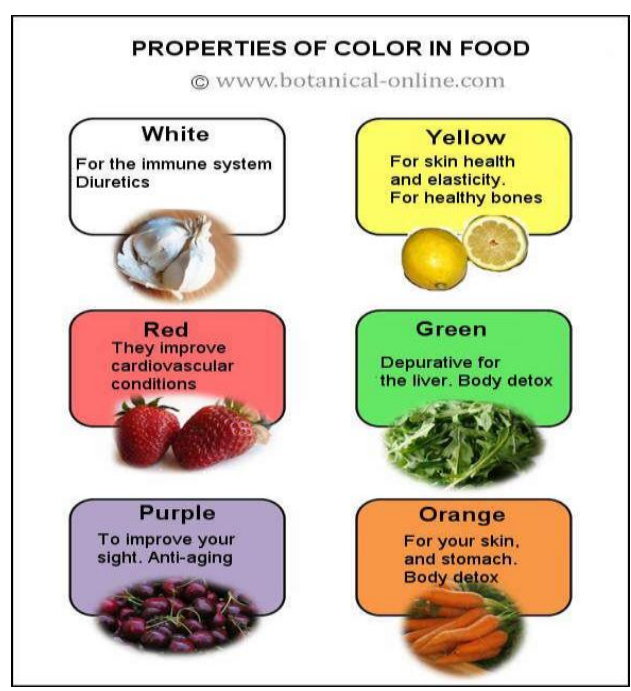
vegetables of various colors also offer other health benefits. As a result nutritionists encourage us to eat 2 to 4 servings of fruits and 3 to 5 serving of vegetables a day. It is important to choose a rainbow of colors. This means picking one daily serving of fruit and vegetable from each of the four color groups - Red orange, yellow, green and purple blue. All those different colors will add plenty of flavors and textures to dishes, making them more healthy, enjoyable and satisfying.

Eating brightly colored fruits and vegetables can keep us fit and healthy and protect against certain cancers, arthritis, heart disease cataracts, and even premature ageing.

Red fruits and vegetables are colored by a natural plant pigment named Lycopene, which is in high concentration in tomatoes, in particular when they are cooked.

It is a powerful source of antioxidant that help reduce the risk of cancer and keep heart healthy. It reduces oxidative stress and hence development of Cancer. The health benefits of eating cooked tomatoes are more significant than supplementing by Lycopene alone. It helps fight urinary tract infection. Other food rich in Lycopene include water melon, apricots and Pink Guava.

Purple/Blue - The distinctive colour of blue/purple in fruits and vegetables is given by plant pigment anthocynins. Anthocynins are strong antioxidants. They have a beneficial impact on obesity control, diabetes, cardiovascular disease and improvement of brain and eye function. Anthocyanins are anti inflammatory, anti carcinogenic and anti microbial. Food rich in Anthocyanins include raspberries, red cherries, straw berries, black currants, black berries, blue berries, and red cabbage.

Orange and yellow fruits and vegetables contain carotenoid. A well known carotenoid called Betacarotene is found in sweet potatoes, pumpkins and carrots. It is converted to vitamin A, which helps maintain healthy mucous membranes and healthy eyes. Another carotene called Lutein is stored in the eye and has been found to prevent cataracts and age related macular degeneration, 
which can leads to blindness. Orange and yellow foods that are rich in carotenoids include: oranges, carrots, sweet potatoes, pumpkin.

Green vegetables contain a range of phytochemicals including carotenoids, indoles and saponins which have anti cancer properties. Green vegetables contain plenty of fibre, minerals, vitamins B, Iron, vitamin $\mathrm{C}$ and $\mathrm{E}$, vitamin $\mathrm{K}$ and Folates.

Green vegetables are green because they contain chlorophyll and masses of other phyto nutrients. Two most researched phyto nutrients are: Lutein \& Glucosinates

Lutein is a type of carotenoids. It is found in very high concentration in the eye and has been associated with lowering risk of eye degeneration associates with ageing and computer exposure, cardiovascular health in particular furring of the arteries and skin ageing. Food rich in lutein are green leafy vegetables - spinach, green beans, peas, broccoli, corn, egg and oranges.

Glucosinates are found in brassicas or cruciferous vegetables. These vegetables are rich in folic acid, cartenoids, selenium vitamin and glucosinates. They are found in broccoli, cabbage and cauliflower.

Blue and purple foods include beet root, blue berries, black berries, black cherries, black raspberries, black currants plums, figs, raisins and pomegranates. This color food contains many different antioxidants such as anthocyanins and reversatrol.

Blue and purple foods are rich in reversatrol - a phyto nutrient which is associated with anti inflamatory and antioxidant properties. Reversatrol may reverse cancer development, protect the cardio vascular system from disease and reduce ageing. It is rich in the skin of black or deep purple grapes and in red wine.

White vegetables especially garlic, onion, leeks and shallots contain a potent phyto nutrient called allicin.

They are known for its antiviral and antibacterial properties. Garlic is a well known antimicrobial food; it has antioxidant, anti cancer properties. It is regularly implicated for cardiovascular health and improved immunity. Onions are also rich in allian, red onion in anthocyanins \& flavonols chemicals for anti inflammatory properties. They are also known to lower risk of blood Clotting. Some members of the white group such as bananas and potatoes, are also a good source of potassium.

Consuming white fruits and vegetables lower the risk of strokes.

We should trust natural color whole foods, and eat as many of those that we know benefits our health. Fruits and vegetables of whole range of colors eaten regularly will lead to benefit from their many healing phyto nutrients.

\section{REFERENCES}

1 Nutrtion Science, B Srilakshmi

2 Clinical Dietetics and Nutrition, F P Antia

3 Nutrition \& Health, The Vegetarian Way, Dr S N Murthy

4 http://www.botanical-online.com/english/importanceofcolorinfood.htm

5 http://english.pravda.ru/health/30-10-2006/85276-color_food-0/

6 http://www.todaysdietitian.com/newarchives/110308p34.shtml

7 http://life.seven-seas.com/the-rainbow-diet-the-importance-of-colour-in-ourfood\#axzz3J6kpyy00

8 http://healthyeating.sfgate.com/colors-fruits-vegetables-play-important-role-1660.html 